\title{
Strategies for targeting the DNA damage response for cancer therapeutics
}

\author{
Dan Zhang ${ }^{1,2}$, Hai-Bo Wang ${ }^{2}$, Kathryn L. Brinkman², Su-Xia Han ${ }^{1}$ and Bo Xu²
}

\begin{abstract}
The DNA damage response is critical for cells to maintain genome stability and survival. In this review, we discuss approaches to targeting critical elements of the DNA damage response for radiosensitization and chemosensitization. In addition, we also discuss strategies for targeting DNA damage response and DNA repair defects in cancer cells for synthetic lethality.
\end{abstract}

Key words DNA damage response, DNA repair, synthetic lethality, PARP inhibitors

Maintaining genomic integrity by protecting against various types of DNA damage is an essential process for all living creatures. Studies of mammalian mechanisms involved in responding to and repairing damaged DNA revealed that there are comprehensive coordinated pathways activated that regulate the DNA damage response (DDR) and DNA repair ${ }^{[1,2]}$. Investigations have demonstrated that cells with atypical expression of prominent proteins involved in these pathways frequently displayed altered sensitivity to DNA damaging agents such as ionizing radiation or many chemotherapeutic drugs ${ }^{[3]}$. These findings led to a rapid interest in exploring targeting DDR proteins (mainly through inhibition) to increase the effectiveness of cancer therapeutics (termed radiosensitization and chemosensitization). While several approaches have been developed successfully in this direction, achieving tumor selectivity remains a critical challenge to the molecular targeted cancer therapy.

\section{DDR and DNA Repair}

The optimal DDR deals with environmental or

Authors' Affiliations: 'Department of Radiation Oncology, The First Affiliated Hospital of Xi'an Jiaotong University Medical School, Xi'an, Shaanxi 710061, P. R. China; 'Department of Radiation Oncology, The Methodist Hospital Research Institute, Houston, TX 77030, USA.

\section{Corresponding Authors:}

Bo Xu, Department of Radiation Oncology, The Methodist Hospital Research Institute, 6670 Bertner Avenue, Houston, TX 77030, USA. Tel: +1-713-441-3996; Fax: +1-713-441-4493; Email: bxu@tmhs.org. Su-Xia Han, Department of Radiation Oncology, First Affiliated Hospital, Xi' an Jiaotong University, Xi'an, Shaanxi 710061, P. R. China. Email: hansuxia22099@yahoo.com.cn.

doi: 10.5732/cjc.012.10087 endogenous insults to DNA to prevent genetic instability and promote survival (Figure 1). Activation of cell cycle checkpoints, which slows down cell cycle progression, is one of the early steps in the DDR. The overall function of cell cycle checkpoints is to provide time for damage detection and appropriate DNA repair ${ }^{[4]}$. With optimal DNA repair, cells are sometimes able to recover from the damage and resume normal cell growth. On the other hand, when the damage is irreparable, programmed cell death is triggered. To deal with various types of DNA damage, the mammalian DNA repair system includes mechanisms such as base excision repair (BER), nucleotide excision repair (NER), mismatch repair (MMR), non-homologous end joining (NHEJ), and homologous recombination $(\mathrm{HR})^{[5]}$. Altogether, the DDR and DNA repair systems are orchestrated by a comprehensive signaling network controlling processes from transcriptional regulation to posttranslational modifications. Several categories of proteins have been extensively studied, including damage sensors, transducers, mediators and effectors ${ }^{[6]}$. Sensors are typically chromatin-bound proteins that recognize DNA damage and recruit transducers. Transducers can amplify damage response signals by posttranslational modifications, such as phosphorylation. Mediators are critical for transducers to recognize downstream effectors and may be critical for the signal on/off switch. Effectors negatively regulate cell cycle progression and might also be critical for activation of DNA repair or apoptosis. Overall, many of these evolutionarily conserved gene products that are critical for DDR and DNA repair have been explored as potential targets for cancer therapy ${ }^{[7]}$. 


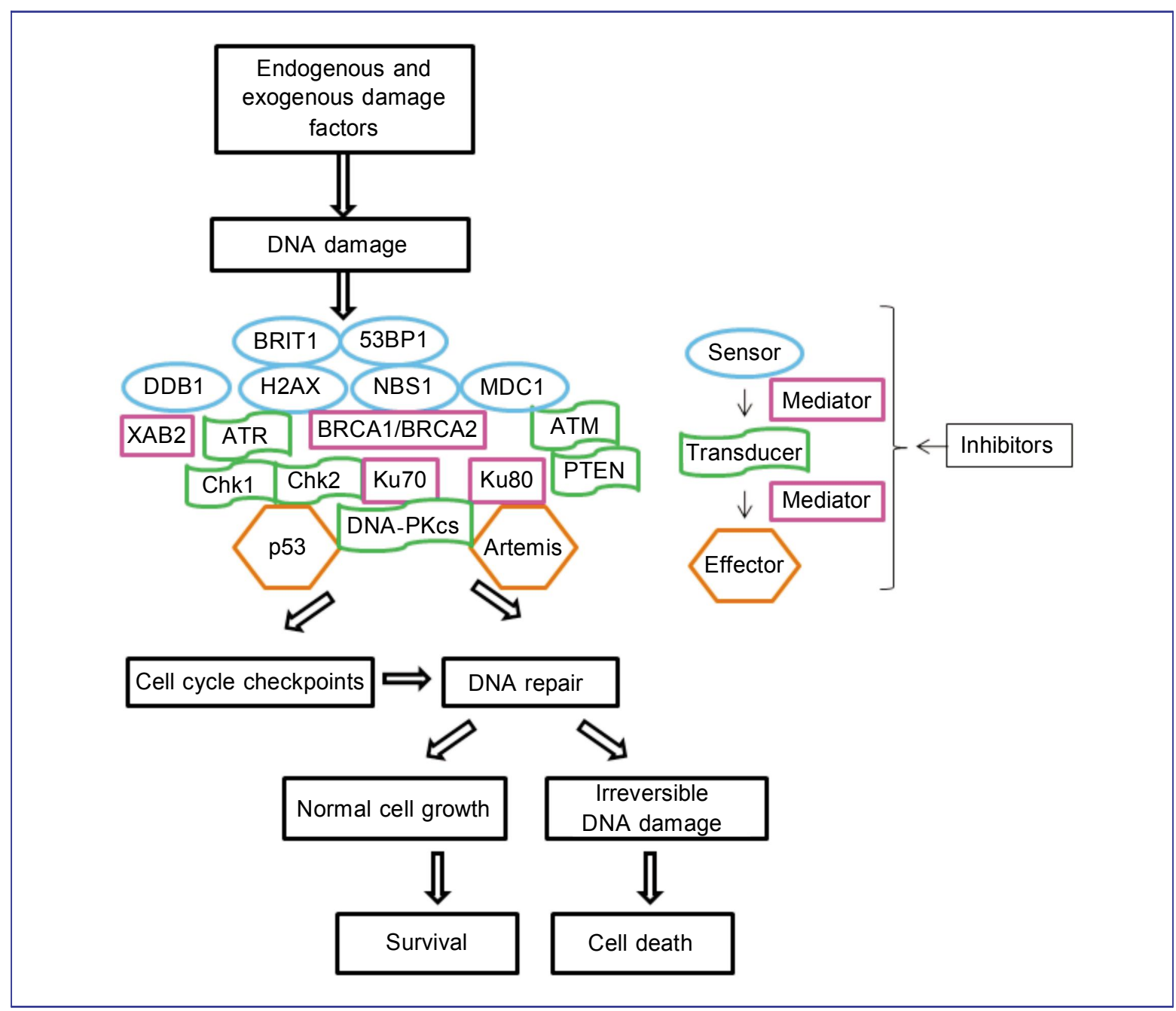

Figure 1. Schematic illustration of the DNA damage response and DNA repair.

\section{Targeting the DDR for Radiosensitiza- tion and Chemosensitization}

Several major kinases that play a critical role in the DDR have been extensively investigated for developing small molecules to enhance sensitivity to radiotherapy and chemotherapy. These druggable kinases include the ataxia telangiectasia mutated (ATM) and DNA-dependent protein kinase catalytic subunit (DNA-PKcs). Well conserved through evolution, ATM and DNA-PKcs are family members of the phosphatidylinositol-3 (PI-3) kinase-related kinase family, and mutation of the ATM kinase causes ataxia telangiectasia manifested by neurodegeneration, cancer, immunodeficiency, and hyper-radiosensitivity ${ }^{[8]}$. Early studies show that $\mathrm{Pl}-3$ kinase inhibitors such as wortmannin and LY294002 can inhibit ATM, the ATM and Rad3-related kinase (ATR), and DNA-PKcs to achieve radiosensitization [9,10]. More specific inhibitors of ATM (KU55933, KU60019, and CP466722) have been developed and showed impressive in vitro activity as radiosensitizers and chemosensitizers ${ }^{[11-13]}$. For DNA-PKcs, small molecules such as Nu7441, IC8736 (flavone-based inhibitor), and SU11752 (ATP competitive inhibitor) have shown promising sensitization effects, despite significant cytotoxicity due to inhibition of the NHEJ process ${ }^{[14-16]}$. The bottleneck of developing radiosensitizers and chemosensitizers is to achieve specificity and selectivity. Since ATM (as well as DNA-PKcs) is a master controller of DDR, determining the specific downstream pathway regulating radiosensitivity is critical for development of inhibitors. In addition to small molecules, small peptides targeting Chk2 kinase activatio ${ }^{[17]}$, the ATM-NBS1 interaction ${ }^{[18]}$, and DNA-PKcs autophosphorylation ${ }^{[19]}$ have been reported to possess radiosensitization activity in 
vitro. These studies used fusion peptides containing a domain for internalization and an interference domain for specific pathway targeting. Although small inhibitory peptides provide specificity for signaling pathways targeted, concerns regarding the stability as well as the potential for the immunogenicity of fusion peptides limit further clinical development.

\section{Targeting DDR for Synthetic Lethality}

A critical challenge for molecular targeted cancer therapy is how to discriminate tumor and normal cells. Traditional chemotherapeutic drugs are cytotoxic because they affect DNA synthesis. Since tumor cells typically have a high proliferation rate, cytotoxic drugs prevail against tumor cells. On the other hand, overexpression of some oncoproteins has been explored for molecular targeted therapy. Synthetic lethality, which is caused by a combination of two genetic mutations that lead to cell death, is one emerging strategy for molecular targeted cancer therapy ${ }^{[20]}$. Since suboptimal DDR and DNA repair are well recognized in cancer cells, it is likely that when one DDR protein has been functionally mutated, an additional functional mutation on a different protein can result in cell death. This concept offers an intriguing strategy for developing anti-cancer therapies.

One example of successfully targeting DNA repair for synthetic lethality is the use of poly (ADPribose)polymerase (PARP) inhibitors in BRCA1/BRCA2-mutant breast cancer or ovarian cancer cells ${ }^{[21,22]}$. BRCA1/ BRCA2 are critical elements in HR-based repair of DNA double strand breaks ${ }^{[]}$. BRCA1 and BRCA2 are frequently mutated in breast cancer and ovarian cancer patients, and mutation of these genes leads to inefficient DNA DSB repair. On the other hand, PARP binds to single-strand breaks (SSBs) and facilitates SSB repair. Inhibition of PARP results in persistent SSBs, resulting in replication-associated DSBs that could be lethal for HR-defective tumors. This makes PARP inhibitors a thousand times more toxic in BRCA-mutant cells. Promising preclinical studies have led to the development of several inhibitors including iniparid, olaparib, PF-01367338, veliparib, and CEP-9722 for clinical trials ${ }^{[7]}$

Using small interfering RNA (siRNA) screening, investigators are also currently working to identify additional HR repair genes that can mediate synthetic lethality with PARP1 inhibition ${ }^{[23]}$. Genes involved in NER (such as $D D B 1$ and $X A B 2$ ) and the tumor suppressor gene $P T E N$ have been found to be synthetically lethal with a PARP inhibitor ${ }^{[23,24]}$. BRIT1, a chromatin-binding protein required for recruitment of many important DDR proteins such as ATM, MDC1, NBS1, RAD51, and BRCA2 to DNA damage sites, also shows synthetic lethality with PARP inhibitors ${ }^{[25,26]}$. In addition, tankyrase 1, another PARP family member involved largely in telomere maintenance, shows synthetic lethality with BRCA1 deficiency ${ }^{[27]}$. A recent study demonstrated that mantle cell lymphoma (MCL) cells deficient in both ATM and p53 are more sensitive to PARP inhibition than cells lacking ATM function alone or their normal counterparts $^{[28]}$.

Despite promising early clinical results ${ }^{[29]}$, iniparib combined with chemotherapy failed to demonstrate any survival improvement in triple-negative metastatic breast cancer patients in a randomized phase III clinical trial (American Society of Clinical Oncology 2011 Annual meeting report). This study raises concerns about the clinical significance of PARP inhibition and has since attracted extensive discussion ${ }^{[30]}$. One major concern is that there are additional, yet unidentified molecular factors that may affect PARP1 inhibitors ${ }^{[28]}$. Synthetic lethality might be compromised because cancers may employ multiple pathways to overcome a defect in one DNA repair pathway ${ }^{[31]}$. More importantly, the tumor specificity of many DNA repair components is not well defined. Since iniparib is less potent than most of other compounds under development, there are concerns whether iniparib should represent a PARP inhibitor in clinical studies. Recent studies have shown that iniparib, which can modify cysteine-containing proteins nonselectively in tumor cells, is not a bona fide inhibitor of PARP, and cautions against comparing clinical trial results using iniparib with other PARP inhibitors ${ }^{[32]}$. Further, another article published afterwards supports the finding that iniparib does not actually inhibit PARP in vitro and concludes that iniparib is not suitable for clinical studies involving PARP inhibition ${ }^{[33]}$. Unrelated to the molecular mechanisms, a concern has been raised that patient "cross-over" in the randomized clinical trial might partially contribute to the negative clinical result ${ }^{[34]}$. Together, these studies indicate that despite the disappointing phase III clinical trials of iniparib, studies should continue to investigate clinical benefits of PARP inhibitors. Further clinical trials using verified, specific inhibitors of PARP as monotherapy and/or in combination with radiotherapy and chemotherapy are greatly anticipated. Studies on the mechanisms of synthetic lethality would help identify critical patient populations that will benefit from the therapy. Meanwhile, further research on identifying inhibitors of other potential DDR 
targets in a variety of tumor tissue types will broaden the applicability of this strategy.

\section{Acknowledgments}

We thank all members of the $\mathrm{Xu}$ laboratory for helpful comments on the manuscript. This work was supported in part by grants from the National Institutes of Health, USA (No. R01CA133093 and R01ES016354) and the National Natural Science Foundation of China (No. 81001027).

Received: 2012-04-06; accepted: 2012-04-20.

\section{References}

[1] Bartek J, Bartkova J, Lukas J. DNA damage signalling guards against activated oncogenes and tumour progression. Oncogene, 2007,26:7773-7779

[2] Jackson SP, Bartek J. The DNA-damage response in human biology and disease. Nature, 2009,461:1071-1078.

[3] Jeggo P, Lavin MF. Cellular radiosensitivity: how much better do we understand it? Int J Radiat Biol, 2009,85:1061-1081.

[4] Kastan MB, Bartek J. Cell-cycle checkpoints and cancer. Nature, 2004,432:316-323.

[5] Jeggo PA. DNA breakage and repair. Adv Genet, 1998,38: 185-218.

[6] Harper JW, Elledge SJ. The DNA damage response: ten years after. Mol Cell, 2007,28:739-745.

[7] Lord CJ, Ashworth A. The DNA damage response and cancer therapy. Nature, 2012,481:287-294

[8] Shiloh Y. ATM and related protein kinases: safeguarding genome integrity. Nat Rev Cancer, 2003,3:155-168.

[9] Sarkaria JN, Tibbetts RS, Busby EC, et al. Inhibition of phosphoinositide 3-kinase related kinases by the radiosensitizing agent wortmannin. Cancer Res, 1998,58:43754382.

[10] Izzard RA, Jackson SP, Smith GC. Competitive and noncompetitive inhibition of the DNA-dependent protein kinase Cancer Res, 1999,59:2581-2586.

[11] Hickson I, Zhao Y, Richardson CJ, et al. Identification and characterization of a novel and specific inhibitor of the ataxiatelangiectasia mutated kinase ATM. Cancer Res, 2004,64: 9152-9159

[12] Golding SE, Rosenberg E, Valerie N, et al. Improved ATM kinase inhibitor KU-60019 radiosensitizes glioma cells, compromises insulin, AKT and ERK prosurvival signaling, and inhibits migration and invasion. Mol Cancer Ther, 2009,8:28942902.

[13] Rainey MD, Charlton ME, Stanton RV, et al. Transient inhibition of ATM kinase is sufficient to enhance cellular sensitivity to ionizing radiation. Cancer Res, 2008,68:7466-7474

[14] Zhao Y, Thomas HD, Batey MA, et al. Preclinical evaluation of a potent novel DNA-dependent protein kinase inhibitor NU7441. Cancer Res, 2006,66:5354-5362.

[15] Shinohara ET, Geng L, Tan J, et al. DNA-dependent protein kinase is a molecular target for the development of noncytotoxic radiation-sensitizing drugs. Cancer Res, 2005,65: 4987-4992.

[16] Ismail IH, Martensson S, Moshinsky D, et al. SU11752 inhibits the DNA-dependent protein kinase and DNA double-strand break repair resulting in ionizing radiation sensitization. Oncogene, 2004,23:873-882.
[17] Suganuma M, Kawabe T, Hori H, et al. Sensitization of cancer cells to DNA damage-induced cell death by specific cell cycle G2 checkpoint abrogation. Cancer Res, 1999,59:5887-5891.

[18] Cariveau MJ, Tang X, Cui XL, et al. Characterization of an NBS1 C-terminal peptide that can inhibit ataxia telangiectasia mutated (ATM)-mediated DNA damage responses and enhance radiosensitivity. Mol Pharmacol, 2007,72:320-326.

[19] Sun X, Yang C, Liu H, et al. Identification and characterization of a small inhibitory peptide that can target DNA-PKcs autophosphorylation and increase tumor radiosensitivity. Int $\mathrm{J}$ Radiat Oncol Biol Phys, 2012. [Epub ahead of print]

[20] Kaelin WG Jr. The concept of synthetic lethality in the context of anticancer therapy. Nat Rev Cancer, 2005,5:689-698.

[21] Bryant HE, Schultz N, Thomas HD, et al. Specific killing of BRCA2-deficient tumours with inhibitors of poly (ADP-ribose) polymerase. Nature, 2005,434:913-917.

[22] Farmer $\mathrm{H}$, McCabe N, Lord CJ, et al. Targeting the DNA repair defect in BRCA mutant cells as a therapeutic strategy. Nature, 2005,434:917-921.

[23] Lord CJ, McDonald S, Swift S, et al. A high-throughput RNA interference screen for DNA repair determinants of PARP inhibitor sensitivity. DNA Repair (Amst), 2008,7:2010-2019.

[24] Mendes-Pereira AM, Martin SA, Brough R, et al. Synthetic lethal targeting of PTEN mutant cells with PARP inhibitors. EMBO Mol Med, 2009,1:315-322.

[25] Peng G, Yim EK, Dai H, et al. BRIT1/MCPH1 links chromatin remodelling to DNA damage response. Nat Cell Biol, 2009,11: 865-872.

[26] Rai R, Phadnis A, Haralkar S, et al. Differential regulation of centrosome integrity by DNA damage response proteins. Cell Cycle, 2008, 7:2225-2233.

[27] McCabe N, Cerone MA, Ohishi T, et al. Targeting Tankyrase 1 as a therapeutic strategy for BRCA-associated cancer. Oncogene, 2009,28:1465-1470.

[28] Williamson CT, Kubota E, Hamill JD, et al. Enhanced cytotoxicity of PARP inhibition in mantle cell lymphoma harbouring mutations in both ATM and p53. EMBO Mol Med, 2012,4:1-13

[29] O'Shaughnessy J, Osborne C, Pippen JE, et al. Iniparib plus chemotherapy in metastatic triple-negative breast cancer. N Engl J Med, 2011,364:205-214.

[30] Guha M. PARP inhibitors stumble in breast cancer. Nat Biotechnol, 2011,29:373-374.

[31] Shaheen M, Allen C, Nickoloff JA, et al. Synthetic lethality: exploiting the addiction of cancer to DNA repair. Blood, 2011,117:6074-6082

[32] Liu X, Shi Y, Maag DX, et al. Iniparib nonselectively modifies 
cysteine-containing proteins in tumor cells and is not a Bona Fide PARP inhibitor. Clin Cancer Res, 2012, 18:510-523.

[33] Patel AG, De Lorenzo SB, Flatten KS, et al. Failure of iniparib to inhibit poly (ADP-ribose) polymerase in vitro. Clin Cancer
Res, 2012,18:1655-1662.

[34] Fojo T, Amiri-Kordestani L, Bates SE. Potential pitfalls of crossover and thoughts on iniparib in triple-negative breast cancer. J Natl Cancer Inst, 2011,103:1738-1740.

\title{
中山大学肿瘤防治中心
}

SUN YAT-SEN UNIVERSITY CANCER CENTER

\section{Sino-French 2012 Conference in Thoracic Oncology}

\section{November 17, 2012}

\author{
23F Conference Room, Sun Yat-sen University Cancer Center \\ 651 Dongfeng East Road, Guangzhou 510060, P. R. China \\ Online registration from September 1 to October 31, 2012 at http://thoracic2012.sysucc.org.cn/
}

\section{Organizing Committee}

Hosting institution:

Sun Yat-sen University Cancer Center, China

Partnering academic institutions:

$10-$ Institute Gustave Roussy (IGR), France

Centre Chirurgical Marie Lannelongue (CCML), France

Jagiellonian University Medical College, Poland

With the support of:

Guangdong Provincial Anticancer Association

\section{Chinese Journal of Cancer}

Consulate General of France in Guangzhou

\section{Meeting Description}

The Sino-French 2012 Conference in Thoracic Oncology is hosted by the Department of Thoracic Surgery in Sun Yat-sen University Cancer Center, a leading comprehensive institution for cancer care, research, education and prevention in South China.

The meeting is also organized in collaboration with two renowned French hospitals, namely, Institute Gustave Roussy (IGR) and Centre Chirurgical Marie Lannelongue (CCML). IGR is the leading European anticancer centre, and bases its uniqueness on therapeutic innovation and development of personalized medicine. CCML is a non-profit organization, with a status of university teaching hospital CCML is specialized in thoracic surgery and interventions (heart, lungs, major vessels, etc.) and has been historically at the cutting edge of medical practice (1956: 1st CPB (cardio-pulmonary bypass) and in 1985: 1st successful Heart-Lung transplantation). The conference will convene international specialists, from both France and China and aims to address various topics in thoracic oncology with a multi-disciplinary approach: surgery, radiation oncology and medical oncology. It will be divided in 3 plenary sessions:

1. Lung Cancer Surgery

2. Innovation in Surgical Techniques

3. Multi-Disciplinary Treatment in Lung Cancer

\section{Conference Honorary Chairs}

- Yi-Xin ZENG, M.D., Ph.D.

Professor and President

Sun Yat-sen University Cancer Center

- Alexander EGGERMONT, M.D., Ph.D.

Professor and General Director

Institute Gustave Roussy

\section{Conference Chairs}

- Tie-Hua RONG, M.D.

Professor, Department of Thoracic Surgery

Sun Yat-sen University Cancer Center

- Thierry LE CHEVALIER, M.D.

Associate Professor,

College of Medicine, Head, Lung Department

Institute Gustave Roussy

- Philippe DARTEVELLE, M.D.

Professor and Director,

Department of Thoracic and Vascular Surgery

Centre Chirurgical Marie Lannelongue

- Lan-Jun ZHANG, M.D. Ph.D.,

Professor and Director,

Department of Thoracic Surgery

Sun Yat-sen University Cancer Center

\section{Secretariat}

Sun Yat-sen University Cancer Center

Address: 651 Dongfeng East Road

Guangzhou 510060, P.R.China

Email: thoracic2012@sysucc.org.cn

Fax: +86-20-8734 3628

Phone: +86-20-8734 3066 (Ms. Shuang Liao, English)

Phone: +86-20-8734 3628 (Ms. Lian-Juan Chen, Chinese) 